\title{
Chirality quantum phase transition in the Dirac oscillator
}

\author{
A. Bermudez, ${ }^{1}$ M. A. Martin-Delgado, ${ }^{1}$ and A. Luis ${ }^{2}$ \\ ${ }^{1}$ Departamento de Física Teórica I, Universidad Complutense, 28040 Madrid, Spain \\ ${ }^{2}$ Departamento de Óptica, Universidad Complutense, 28040 Madrid, Spain
}

(Received 1 February 2008; published 11 June 2008)

\begin{abstract}
We study a relativistic spin-1/2 fermion subjected to a Dirac oscillator coupling and a constant magnetic field. An interplay between opposed chirality interactions culminates in the appearance of a relativistic quantum phase transition, which can be fully characterized. We obtain analytical expressions for the energy gap, order parameter, and canonical quantum fluctuations across the critical point. Moreover, we also discuss the effect of this phase transition on the statistics of the chiral bosonic ensemble, where its super- or subPoissonian nature can be controlled by means of external parameters. Finally, we study the entanglement properties between the degrees of freedom in the relativistic ground state, where an interesting transition between a biseparable and a genuinely tripartite entangled state occurs.
\end{abstract}

DOI: 10.1103/PhysRevA.77.063815

PACS number(s): 42.50.Lc, 42.50.Ar, 42.50.Dv

\section{INTRODUCTION}

Classical phase transitions describe an abrupt change in the physical properties of a system caused by the modification of its temperature. This phenomenon usually entails a change in the symmetry of the phases involved, and is commonly driven by thermal fluctuations. Consequently, classical phase transition cannot persist at zero temperature where the absence of thermal fluctuations forbids the sudden change of phase. Nonetheless, other kinds of fluctuations exist at zero temperature, the so-called quantum fluctuations, which can also be responsible for a dramatic change in the properties of the system. In this case, the change is driven by the modification of certain couplings that describe the interactions between the microscopic constituents of the system, and is usually known as a quantum phase transition [1].

These critical phenomena arise in the thermodynamical limit of certain many-body systems, where the number of particles $N \rightarrow \infty$. Usually, the description of such systems is extremely complex and one must deal with numerical methods. Nevertheless, there exist certain situations where a simplified model, which can be exactly solved, captures the full essence of the problem displaying such an abrupt change of the system properties. For instance, a collection of $N$ twolevel atoms interacting with a single mode of the radiation field, known as the Dicke model, displays a quantum phase transition whose features can be justified by means of a simple two-mode Hamiltonian in the thermodynamical limit $[2,3]$. Other two-mode Hamiltonians which also display critical phenomena have been studied in the field of quantum optics $[4,5]$ or in nuclear physics [6].

In this work, we shall be concerned with a relativistic toy model that involves two phonon modes and also displays a quantum phase transition. This critical phenomenon occurs in a fermionic relativistic harmonic oscillator, also known as a Dirac oscillator [7,8], when an additional constant magnetic field is applied. This relativistic fermion of mass $m$ and charge $-e$ is described by the following Dirac equation:

$$
i \hbar \frac{\partial|\Psi\rangle}{\partial t}=\left[c \boldsymbol{\alpha}(\mathbf{p}-i m \beta \omega \mathbf{r}+e \mathbf{A})+\beta m c^{2}\right]|\Psi\rangle,
$$

where $|\Psi\rangle$ stands for the Dirac four-component spinor, $\mathbf{r}$ and p represent the position and momentum operators, $\omega$ is the
Dirac oscillator frequency, $c$ stands for the speed of light and $\beta:=\operatorname{diag}(\mathbb{I},-\mathbb{I}), \alpha_{j}:=\operatorname{off}-\operatorname{diag}\left(\sigma_{j}, \sigma_{j}\right)$ are the Dirac matrices related to the usual Pauli matrices [9]. Here, the magnetic field is introduced by minimal coupling $\mathbf{p} \rightarrow \mathbf{p}+e \mathbf{A}$, where $\mathbf{A}$ is the vector potential related to the magnetic field through $\mathbf{B}=\nabla \wedge \mathbf{A}$. On the other hand, the Dirac oscillator coupling is introduced by a nonminimal coupling $\mathbf{p}-\mathrm{i} m \beta \omega \mathbf{r}$, where $\omega$ stands for the Dirac oscillator frequency.

Here, we shall focus on a two-dimensional setup where the Dirac matrices become the well-known Pauli matrices $\alpha_{x}=\sigma_{x}, \alpha_{y}=\sigma_{y}, \beta=\sigma_{z}$. In this scenario, Eq. (1) can be expressed as

$$
i \hbar \frac{\partial|\Psi\rangle}{\partial t}=\left[c \sum_{j=1}^{2} \sigma_{j}\left(p_{j}-\mathrm{i} m \beta \sigma_{z} x_{j}+e A_{j}\right)+\sigma_{z} m c^{2}\right]|\Psi\rangle,
$$

where $|\Psi\rangle$ is a two-component spinor which mixes spin-up and -down components with positive and negative energies. Remarkably enough, the Dirac oscillator coupling endows the particle with an intrinsic left-handed chirality which is only present in this two-dimensional scenario [10]. Conversely, the magnetic field coupling favors a right-handed chirality [11], and therefore an intriguing interplay is set up. This system can be considered as a relativistic extension of chiral harmonic oscillators, which carry dual aspects of a certain symmetry (i.e., chirality in the plane). Such nonrelativistic systems have been studied from a fundamental point of view [12], and an interesting connection to topological Chern-Simons gauge theories has also been pointed out $[13,14]$.

In this paper, we show how this relativistic chiral oscillator presents several intriguing critical properties, and offer an ideal scenario where to study the effect of opposed chirality interactions in a two-dimensional setting. In Sec. II, we show how the relativistic Hamiltonian in Eq. (2) can be exactly mapped onto a pair of simultaneous Jaynes-Cummings (JC) and anti-Jaynes-Cummings (AJC) interactions $[15,16]$ with right- and left-handed chirality, respectively. This result differs substantially from previous situations where only a distinctive chiral interaction appeared $[10,11]$, and opens up the 
possibility to study a unique interplay between left- and right-handed interactions. In Sec. III, we describe two limiting cases: a weak magnetic field regime where the system displays a remarkable left-handed chirality, and a strong magnetic field regime with an opposite right-handed chirality. For intermediate couplings, we obtain the complete energy spectrum and the associated eigenstates in Sec. IV. To accomplish such task, we perform a unitary transformation which turns the Hamiltonian into a single-mode interaction which can be easily diagonalized. This exact solution shows how an unusual competition between chiral terms arises, and leads to a relativistic level crossing phenomenon for a critical value of the JC and AJC couplings, which is described in detail in Sec. V. In this sense, several analogies with second order quantum phase transitions occur, such as the energy gap suppression, the divergence of quantum fluctuations, and the existence of an order parameter that reveals the existence of a quantum phase transition. Furthermore, we also show that the statistical nature of the chiral phonon distribution displays an abrupt change across the critical point, where super-Poissonian chiral phonons turn into sub-Poissonian phonons and vice versa. Finally, in the Appendix, we give some details for the construction of the system eigenstates, which are closely related to $\mathrm{SU}(1,1)$ coherent states.

\section{EXACT MAPPING ONTO A SIMULTANEOUS JC-AJC HAMILTONIAN}

Let us first provide the exact mapping onto a pair of simultaneous JC and AJC couplings with opposite chiralities. We shall work in the axial gauge, where a constant magnetic field $\mathbf{B}=B \mathbf{e}_{z}$ is described by the following vector potential $\mathbf{A}:=\frac{B}{2}[-y, x, 0]$. In this setup, the dynamics of a relativistic fermion is described by two different frequencies, the Dirac oscillator frequency $\omega$ and the cyclotron frequency $\omega_{c}$ : $=e B / \mathrm{m}$. In this regard, we must introduce a pair of sets of creation-annihilation operators

$$
\begin{aligned}
& a_{i}=\frac{1}{\sqrt{2}}\left(\frac{1}{\Delta} r_{i}+i \frac{\Delta}{\hbar} p_{i}\right), \quad a_{i}^{\dagger}=\frac{1}{\sqrt{2}}\left(\frac{1}{\Delta} r_{i}-i \frac{\Delta}{\hbar} p_{i}\right), \\
& \tilde{a}_{i}=\frac{1}{\sqrt{2}}\left(\frac{1}{\widetilde{\Delta}} r_{i}+i \frac{\tilde{\Delta}}{\hbar} p_{i}\right), \quad \tilde{a}_{i}^{\dagger}=\frac{1}{\sqrt{2}}\left(\frac{1}{\widetilde{\Delta}} r_{i}-i \frac{\tilde{\Delta}}{\hbar} p_{i}\right),
\end{aligned}
$$

associated to the frequencies $\omega$ and $\widetilde{\omega}:=\omega_{c} / 2$, respectively. Here, $\Delta:=\sqrt{\hbar / m \omega}$ and $\widetilde{\Delta}:=\sqrt{\hbar / m \widetilde{\omega}}$ represent the oscillator's ground-state width associated to each frequency, and we have introduced $i=x, y$ to account for the two possible directions of motion [17]. The substitution of these operators in Eq. (2), followed by the introduction of the chiral annihilation operators for each frequency:

$$
\tilde{a}_{r}:=\frac{1}{\sqrt{2}}\left(\tilde{a}_{x}-i \widetilde{a}_{y}\right), \quad \tilde{a}_{l}:=\frac{1}{\sqrt{2}}\left(\tilde{a}_{x}+i \tilde{a}_{y}\right),
$$

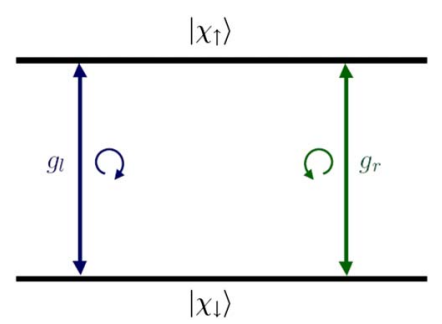

FIG. 1. (Color online) Level scheme representation of the relativistic interaction, which gives rise to spin-flip transitions accompanied by the simultaneous creation and annihilation of chiral phonons. Note that there are two different spin-flip channels depending on the phonon chirality. Namely, the right-handed channel $\left|\chi_{\downarrow}\right\rangle\left|\tilde{n}_{r}+1\right\rangle \leftrightarrow\left|\chi_{\text {row }}\right\rangle\left|\tilde{n}_{r}\right\rangle$, and the left-handed channel $\left|\chi_{\downarrow}\right\rangle\left|n_{l}\right\rangle \leftrightarrow\left|\chi_{\uparrow}\right\rangle\left|n_{l}+1\right\rangle$. Here we have introduced the Pauli spinors $\left|\chi_{\uparrow}\right\rangle:=(1,0)^{t}$ and $\left|\chi_{\downarrow}\right\rangle:=(0,1)^{t}$, and the left-handed $\left|n_{l}\right\rangle$ : $=\left(n_{l} !\right)^{-1 / 2}\left(a_{l}^{\dagger}\right)^{n_{l}}|\mathrm{vac}\rangle$ and right handed $\left|\widetilde{n}_{r}\right\rangle:=\left(\widetilde{n}_{r} !\right)^{-1 / 2}\left(\widetilde{a}_{r}^{\dagger}\right)^{\tilde{n}_{r}}|\mathrm{vac}\rangle$ chiral Fock states.

$$
a_{r}:=\frac{1}{\sqrt{2}}\left(a_{x}-i a_{y}\right), \quad a_{l}:=\frac{1}{\sqrt{2}}\left(a_{x}+i a_{y}\right),
$$

and the consequent creation operators $\tilde{a}_{r}^{\dagger}, \tilde{a}_{l}^{\dagger}, a_{r}^{\dagger}, a_{r}^{\dagger}$, leads to the following bichromatic relativistic Hamiltonian:

$$
H=m c^{2}\left[\begin{array}{cc}
1 & i \sqrt{2 \xi} a_{l}^{\dagger}-i \sqrt{2 \widetilde{\xi}} \widetilde{a}_{r} \\
-i \sqrt{2 \xi} a_{l}+i \sqrt{2 \widetilde{\xi}} \widetilde{a}_{r}^{\dagger} & -1
\end{array}\right],
$$

where the two important parameters $\xi:=\hbar \omega / m c^{2}$ and $\widetilde{\xi}$ : $=\hbar \tilde{\omega} / m c^{2}$ represent the strength of the oscillator and magnetic field coupling with respect to the particle rest mass energy, respectively. This Hamiltonian in Eq. (5) can be rewritten as simultaneous JC and AJC interactions with opposite chiralities

$$
H=\delta \sigma_{z}-H_{\mathrm{JC}}^{\circlearrowleft}\left(g_{r}\right)+H_{\mathrm{AJC}}^{\circlearrowleft}\left(g_{l}\right),
$$

where $\delta:=m c^{2}$ stands for the detuning parameter proportional to the rest mass energy, $H_{\mathrm{JC}}\left(g_{r}\right)$ represents a righthanded Jaynes-Cummings Hamiltonian

$$
H_{\mathrm{JC}}^{\circlearrowleft}\left(g_{r}\right)=g_{r} \sigma^{+} \tilde{a}_{r}+g_{r}^{*} \sigma^{-} \tilde{a}_{r}^{\dagger},
$$

with $g_{r}:=i m c^{2} \sqrt{2 \widetilde{\xi}}$ as the interaction coupling strength. Analogously, the term $H_{\mathrm{AJC}}\left(g_{l}\right)$ stands for a left-handed AntiJaynes-Cummings interaction

$$
H_{\mathrm{AJC}}^{\circlearrowleft}\left(g_{l}\right)=g_{l} \sigma^{+} a_{l}^{\dagger}+g_{l}^{*} \sigma^{-} a_{l},
$$

with a similar coupling strength $g_{l}:=\mathrm{i} m c^{2} \sqrt{2 \xi}$. The relativistic Hamiltonian in Eq. (6) is depicted in Fig. 1, where the JC and AJC couplings give rise to a pair of channels through which the relativistic particle can perform spin-flip transitions.

\section{WEAK AND STRONG MAGNETIC FIELD REGIMES}

The exact mapping of this relativistic model onto a simultaneous JC and AJC couplings allows a neat description of the interaction in terms of a energy level scheme (see Fig. 1). 
The presence of the pair of simultaneous couplings $H_{\mathrm{AJC}}\left(g_{l}\right)$, and $H_{\mathrm{JC}}\left(g_{r}\right)$ in Eq. (6), forbids a straightforward solution of the complete Hamiltonian by analogy to JC-like solutions $[10,11]$. Nonetheless, these techniques can be used to obtain the energy spectrum and corresponding eigenstates in two limiting regimes.

\section{A. Weak magnetic field $\tilde{\xi} \ll \xi$}

In this regime, the Hamiltonian in Eq. (5) becomes

$$
H^{w}=2 \delta \sigma_{z}+g_{l} \sigma^{+} a_{l}^{\dagger}+g_{l}^{*} \sigma^{-} a_{l}+O(\widetilde{\xi} / \xi),
$$

and we can obtain a first approximation to the energy spectrum neglecting corrections of order $O(\tilde{\xi} / \xi)$. Consequently, the relativistic Hamiltonian can be approximately described by a left-handed AJC coupling which can be directly solved [18]. The energy spectrum becomes $E^{w}= \pm E_{n_{l}}^{w}= \pm m c^{2}[1$ $\left.+2 \xi\left(n_{l}+1\right)\right]^{1 / 2}$, where $n_{l}=0,1, \ldots$, represents the number of left-handed phonons. The corresponding eigenstates form an AJC doublet which can be described as

$$
\left| \pm E_{n_{l}}^{w}\right\rangle=C_{n_{l}, \pm}^{w}\left|n_{l}+1\right\rangle\left|\chi_{\uparrow}\right\rangle \mp i C_{n_{l}, \mp}^{w}\left|n_{l}\right\rangle\left|\chi_{\downarrow}\right\rangle,
$$

where we have introduced the set of left-handed Fock states $\left|n_{l}\right\rangle:=\left(n_{l} !\right)^{-1 / 2}\left(a_{l}^{\dagger}\right)^{n_{l}}|\operatorname{vac}\rangle, \quad\left|\chi_{\uparrow}\right\rangle:=(1,0)^{t} \quad$ and $\quad\left|\chi_{\downarrow}\right\rangle:=(0,1)^{t}$ stand for up and down Pauli spinors, and certain normalization constants $C_{n_{l}, \pm}^{w}:=\left[\left(E_{n_{l}}^{w} \pm m c^{2}\right) / 2 E_{n_{l}}^{w}\right]^{1 / 2}$.

\section{B. Strong magnetic field $\tilde{\xi} \gg \xi$}

In this limit, the Hamiltonian in Eq. (5) becomes

$$
H^{s}=2 \delta \sigma_{z}-g_{r} \sigma^{+} \tilde{a}_{r}-g_{r}^{*} \sigma^{-} \tilde{a}_{r}^{\dagger}+O(\xi / \widetilde{\xi}),
$$

and we can find the energy spectrum up to $O(\xi / \widetilde{\xi})$, which becomes $E^{s}= \pm E_{\tilde{n}_{r}}^{s}= \pm m c^{2}\left[1+2 \widetilde{\xi}\left(\tilde{n}_{r}+1\right)\right]^{1 / 2}$, where $\tilde{n}_{r}$ $=0,1, \ldots$, is the number of right-handed phonons. The corresponding eigenstates form a JC doublet [18] with the following structure:

$$
\left| \pm E_{\tilde{n}_{r}}^{s}\right\rangle=C_{\tilde{n}_{r}, \pm}^{S}\left|\tilde{n}_{r}\right\rangle\left|\chi_{\uparrow}\right\rangle \mp i C_{\tilde{n}_{r}, \mp}^{s}\left|\tilde{n}_{r}+1\right\rangle\left|\chi_{\downarrow}\right\rangle,
$$

with $\left|\tilde{n}_{r}\right\rangle:=\left(\tilde{n}_{r} !\right)^{-1 / 2}\left(\tilde{a}_{r}^{\dagger}\right)^{\tilde{n}_{r}} \mid$ vac $\rangle$ as right-handed Fock states and $C_{\widetilde{n}_{r}, \pm}^{s}:=\left[\left(E_{\tilde{n}_{r}}^{s} \pm m c^{2}\right) / 2 E_{\widetilde{n}_{r}}^{s}\right]^{1 / 2}$.

The latter results can be understood as follows, in the regime where $\tilde{\xi} / \xi \rightarrow 0$, the relativistic system is found in a phase with left chirality. Conversely, in the limit where $\tilde{\xi} / \xi$ $\rightarrow \infty$, a notorious right-handed chiral phase arises. Therefore, a modification of the coupling strengths $\xi, \widetilde{\xi}$ leads to a change of the chiral symmetry, which can be interpreted in the language of quantum phase transitions. We will consider a zero-temperature setup, where thermal fluctuations do not exist and the chirality transition can only be driven by quantum fluctuations. As we show below, the complete relativistic energy spectrum can be obtained for all possible couplings $\xi, \widetilde{\xi}$. This spectrum presents two crucial properties which resemble the usual setting in quantum phase transitions: the energy spectrum is nonanalytical for a critical coupling $(\tilde{\xi} / \xi)_{c}=1$, and the system becomes gapless at this critical point $\Delta E \rightarrow 0$ when $\left|(\tilde{\xi} / \xi)-(\widetilde{\xi} / \xi)_{c}\right| \rightarrow 0$.

\section{EXACT SOLUTION: ENERGY SPECTRUM AND ASSOCIATED EIGENSTATES}

The particular structure of the Hamiltonian in Eq. (5) suggests a different description where the spinorial degrees of freedom are coupled to a unique bosonic operator $b \propto\left(a_{l}\right.$ $\left.-(\tilde{\xi} / \xi)^{1 / 2} \widetilde{a}_{r}^{\dagger}\right)$ which mixes both chiralities depending on the relative coupling strength $\tilde{\xi} / \xi$. In this regard, we derive a unitary transformation which converts the bichromatic Hamiltonian in Eq. (6) into a monochromatic JC (AJC) term that involves such bosonic degree of freedom $b$ with a certain chirality that depends on external parameters $\xi>\widetilde{\xi}(\xi$ $<\widetilde{\xi}$ ). This transformation, which turns out to be a two-mode squeezing operator, allows an insightful derivation of the energy spectrum and its associated eigenstates whose properties depend strongly on the magnitude of the magnetic field applied. As we shall discuss, certain properties of the system, such as chirality, squeezing, phonon statistics, and entanglement, are conditioned to the value of the magnetic field. Moreover, as the magnetic field is varied, a sudden quantum phase transition occurs at $\xi=\widetilde{\xi}$, where both chiralities contribute identically, and the critical theory becomes that of a relativistic free fermion.

\section{A. Left-handed regime $\tilde{\xi}<\xi$}

Under these circumstances, the relativistic Hamiltonian in Eqs. (6)-(8) can be unitarily mapped onto a single-mode anti-Jaynes-Cummings Hamiltonian by means of the following unitary transformation:

$$
U_{\alpha}:=e^{\alpha\left(a_{l} \widetilde{a}_{r}-\widetilde{a}_{r}^{\dagger} a_{l}^{\dagger}\right)},
$$

where the real parameter $\alpha$ depends on the relative strength of the oscillator and magnetic field couplings

$$
\alpha:=\frac{1}{\lambda} \operatorname{arctanh}\left(\frac{\lambda \sqrt{\widetilde{\omega}}}{\sqrt{\omega}-\mu \sqrt{\widetilde{\omega}}}\right),
$$

and we have introduced $\mu:=(\Delta / \widetilde{\Delta}-\widetilde{\Delta} / \Delta) / 2$ and $\lambda$ $:=\sqrt{\mu^{2}+1}$. The transformation laws for the chiral operators under the unitary in Eq. (13) can be described as follows:

$$
\begin{aligned}
& U_{\alpha}^{\dagger} a_{l} U_{\alpha}=\left(\cosh (\lambda \alpha)+\frac{\mu}{\lambda} \sinh (\lambda \alpha)\right) a_{l}-\frac{1}{\lambda} \sinh (\lambda \alpha) \tilde{a}_{r}^{\dagger}, \\
& U_{\alpha}^{\dagger} \widetilde{a}_{r} U_{\alpha}=\left(\cosh (\lambda \alpha)-\frac{\mu}{\lambda} \sinh (\lambda \alpha)\right) \tilde{a}_{r}-\frac{1}{\lambda} \sinh (\lambda \alpha) a_{l}^{\dagger},
\end{aligned}
$$

which lead to the following transformation of the complete original Hamiltonian into a single-mode AJC term

$$
H_{\mathrm{AJC}}^{\circlearrowleft}\left(g_{l}^{\prime}\right):=U_{\alpha} H U_{\alpha}^{\dagger}=\delta \sigma_{z}+g_{l}^{\prime} \sigma^{+} a_{l}^{\dagger}+\left(g_{l}^{\prime}\right)^{*} \sigma^{-} a_{l},
$$

with a modified coupling strength $g_{l}^{\prime}:=i m c^{2} \sqrt{2 \zeta_{l}}$, where $\zeta_{l}(\xi, \widetilde{\xi}):=\xi-\widetilde{\xi}-2 \mu(\widetilde{\xi} \xi)^{1 / 2}$ is related to the initial relevant pa- 
rameters. Once the single-mode Hamiltonian in Eq. (16) has been obtained, we can calculate the energy spectrum and corresponding eigenstates of the original Hamiltonian by solving the single mode coupling. Two Hermitian operators related through a unitary transformation share a common spectrum, and therefore the energy eigenvalues of the Hamiltonian in Eq. (6) can be obtained in analogy to the weak magnetic field regime

$$
E= \pm E_{n_{l}}= \pm m c^{2} \sqrt{1+2 \zeta_{l}(\xi, \widetilde{\xi})\left(n_{l}+1\right)},
$$

where $n_{l}=0,1, \ldots$, represents the number of left-handed quanta. The associated eigenstates are obtained by means of the unitary transform applied to the single-mode left-handed eigenstates

$$
\left| \pm E_{n_{l}}\right\rangle=U_{\alpha}^{\dagger}\left(C_{n_{l}, \pm}\left|n_{l}+1\right\rangle\left|\chi_{\uparrow}\right\rangle \mp i C_{n_{l}, \mp}\left|n_{l}\right\rangle\left|\chi_{\downarrow}\right\rangle\right),
$$

where the normalization constants depend on the energies $C_{n_{l}, \pm}:=\left[\left(E_{n_{l}} \pm m c^{2}\right) / 2 E_{n_{l}}\right]^{1 / 2}$. The transformation in Eq. (13) can be rewritten in the monochromatic scenario as

$$
U_{\alpha}=e^{-(\alpha \tilde{\mu} / 2)\left(-a_{r} a_{l}+a_{r}^{\dagger} a_{l}^{\dagger}\right)}
$$

where $\tilde{\mu}:=(\Delta / \widetilde{\Delta}+\tilde{\Delta} / \Delta) / 2$. This transformation can be immediately related to a two-mode squeezing operator in the context of quantum optics with squeezing parameter $z$ $:=-\alpha \tilde{\mu} / 2 \in \mathbb{R}[19]$. The action of such an squeezing operator (19) over left-handed chiral Fock states gives rise to $\mathrm{SU}(1,1)$ coherent states $\left|z, n_{l}\right\rangle:=U_{\alpha}^{\dagger}\left|n_{l}\right\rangle|\mathrm{vac}\rangle_{r}$ [36] (see the Appendix for some details)

$$
\left|z, n_{l}\right\rangle=\mathcal{N}_{n_{l}} \sum_{m=0}^{\infty} \sqrt{\frac{\left(m+n_{l}\right) !}{n_{l} ! m !}}(-1)^{m} \tanh ^{m}|z|\left|m+n_{l}, m\right\rangle,
$$

where we have introduced the normalization constant $\mathcal{N}_{n_{l}}$ $:=\cosh ^{-\left(n_{l}+1\right)}|z|$. Since right-handed operators are not present in the effective Hamiltonian of Eq. (16), and consequently do not participate in the relativistic dynamics, we have chosen the right-handed vacuum for simplicity. The state corresponding to $n_{l}=0$ can be identified with the two-mode squeezed vacuum state, an archetypical state in the field of quantum optics that becomes highly nonclassical for a large squeezing parameter. Therefore, the energy eigenstates of the relativistic fermion in Eq. (18) can be expressed as

$$
\left| \pm E_{n_{l}}\right\rangle=C_{n_{l}, \pm}\left|z, n_{l}+1\right\rangle\left|\chi_{\uparrow}\right\rangle \mp i C_{n_{l}, \mp}\left|z, n_{l}\right\rangle\left|\chi_{\downarrow}\right\rangle,
$$

which is identical to an AJC-like doublet where $\mathrm{SU}(1,1)$ coherent states are entangled with the relativistic spinors. Such a state possesses remarkable nonclassical properties, such as spin-orbit entanglement or sub-Poissonian statistics. With respect to the weak magnetic field eigenstates in Eqs. (10), these states present certain novel features, such as an intermode chiral entanglement, or bosonic statistics which depend on the coupling strength $\widetilde{\xi} / \xi$. All these interesting properties will be described in detail in forthcoming sections. We shall be also interested in the properties of the fermionic ground state $E_{g}=m c^{2}$

$$
|g\rangle=|z, 0\rangle\left|\chi_{\uparrow}\right\rangle=\frac{1}{\cosh |z|} \sum_{m=0}^{\infty}(-1)^{m} \tanh ^{m}|z||m, m\rangle\left|\chi_{\uparrow}\right\rangle,
$$

which can be notably interpreted as a spin-up squeezed vacuum state, where the squeezing parameter $z=-\alpha \tilde{\mu} / 2 \mathrm{de}$ pends on the relative coupling strengths $\xi, \widetilde{\xi}$.

\section{B. Right-handed regime $\widetilde{\xi}>\xi$}

In this case, the original Hamiltonian in Eqs. (6)-(8) is transformed into a Jaynes-Cummings Hamiltonian under the action of

$$
U_{\tilde{\alpha}^{:}}:=e^{\widetilde{\alpha}\left(a_{l} \tilde{a}_{r}-\widetilde{a}_{r}^{\dagger} a_{l}^{\dagger}\right)}
$$

where the parameter $\tilde{\alpha}$ in this regime becomes

$$
\tilde{\alpha}:=\frac{1}{\lambda} \operatorname{arctanh}\left(\frac{\lambda \sqrt{\omega}}{\sqrt{\widetilde{\omega}}+\mu \sqrt{\omega}}\right) .
$$

The chiral operators are once again transformed according to Eqs. (15) when the substitution $\alpha \rightarrow \widetilde{\alpha}$ is performed. In this situation, the transformed Hamiltonian becomes a singlemode Jaynes-Cummings term

$$
H_{\mathrm{JC}}^{\circlearrowleft}\left(g_{r}^{\prime}\right):=U_{\widetilde{\alpha}} H U_{\widetilde{\alpha}}^{\dagger}=\delta \sigma_{z}+g_{r}^{\prime} \sigma^{+} a_{r}+\left(g_{r}^{\prime}\right)^{*} \sigma^{-} a_{r}^{\dagger},
$$

where the new coupling strength is $g_{r}^{\prime}:=i m c^{2} \sqrt{2 \zeta_{r}}$, and $\zeta_{r}(\xi, \widetilde{\xi}):=\widetilde{\xi}-\xi+2 \mu(\tilde{\xi} \xi)^{1 / 2}$. Analogously to the left-handed regime $\widetilde{\xi}<\xi$, we obtain a single-mode Hamiltonian which can be easily diagonalized following the same procedure as in the strong magnetic field limit, and provides the solution to the complete bichromatic interaction in Eq. (6). In this sense, the energy spectrum becomes

$$
\widetilde{E}= \pm \widetilde{E}_{\widetilde{n}_{r}}= \pm m c^{2} \sqrt{1+2 \zeta_{r}(\xi, \widetilde{\xi})\left(\widetilde{n}_{r}+1\right)}
$$

where $\tilde{n}_{r}=0,1, \ldots$, represents the number of right-handed quanta. Applying the unitary transform in Eq. (23) to the single-mode eigenstates, one obtains the corresponding eigenstates of the complete original Hamiltonian

$$
\left| \pm \widetilde{E}_{\widetilde{n}_{r}}\right\rangle=U_{\widetilde{\alpha}}^{\dagger}\left(\widetilde{C}_{\widetilde{n}_{r}, \pm}\left|\tilde{n}_{r}\right\rangle\left|\chi_{\uparrow}\right\rangle \mp i \widetilde{C}_{\widetilde{n}_{r}, \mp}\left|\tilde{n}_{r}+1\right\rangle\left|\chi_{\downarrow}\right\rangle\right),
$$

where $\widetilde{C}_{\widetilde{n}_{r}, \pm}:=\left[\left(\widetilde{E}_{\widetilde{n}_{r}} \pm m c^{2}\right) / 2 \widetilde{E}_{\widetilde{n}_{r}}\right]^{1 / 2}$. In this regime, the transformation in Eq. (23) in the single-frequency domain becomes

$$
U_{\widetilde{\alpha}}=e^{-(\widetilde{\alpha} \tilde{\mu} / 2)\left(-\widetilde{a}_{r} \widetilde{a}_{l}+\tilde{a}_{r}^{\dagger} \widetilde{a}_{l}^{\dagger}\right)},
$$

which can be reinterpreted once more as a two-mode squeezing operator with a different squeezing parameter $\tilde{z}:=-\tilde{\alpha} \tilde{\mu} / 2 \in \mathbb{R}$. This operator transforms the right-handed chiral Fock states $\left|\tilde{n}_{r}\right\rangle$ into $\mathrm{SU}(1,1)$ coherent states $\left|\widetilde{z}, \tilde{n}_{r}\right\rangle$ $:=U_{\tilde{\alpha}}^{\dagger}|\operatorname{vac}\rangle_{l}\left|\tilde{n}_{r}\right\rangle$, where we have chosen the left-handed vacuum for simplicity (see the Appendix) 


$$
\left|\tilde{z}, \tilde{n}_{r}\right\rangle=\mathcal{N}_{\tilde{n}_{r}} \sum_{\tilde{m}=0}^{\infty} \sqrt{\frac{\left(\tilde{m}+\tilde{n}_{r}\right) !}{\tilde{n}_{r} ! \tilde{m} !}}(-1)^{\tilde{m}} \tanh ^{\tilde{m}}|\tilde{z}|\left|\tilde{m}+\tilde{n}_{r}, \tilde{m}\right\rangle,
$$

where we have introduced the following normalization constant $\mathcal{N}_{\tilde{n}_{r}}:=\cosh ^{-\left(\widetilde{n}_{r}+1\right)}|\bar{z}|$. Such coherent states in Eq. (29) appear in doublets in the fermionic eigenstates of Eq. (22)

$$
\left| \pm \widetilde{E}_{\tilde{n}_{r}}\right\rangle=\widetilde{C}_{\tilde{n}_{r} \pm}\left|\tilde{z}, \tilde{n}_{r}\right\rangle\left|\chi_{\uparrow}\right\rangle \mp i \widetilde{C}_{\tilde{n}_{r}, \mp}\left|\tilde{z}, \tilde{n}_{r}+1\right\rangle\left|\chi_{\downarrow}\right\rangle .
$$

Once again, one can identify these eigenstates as JC-like doublets where $\mathrm{SU}(1,1)$ coherent states become entangled with the relativistic spin degree of freedom. Conversely to the fermionic ground state of the left-handed regime in Eq. (22), the ground state in this regime $E_{\tilde{g}}=m c^{2} \sqrt{1+2 \zeta_{r}}$, cannot be expressed solely by the two-mode squeezed vacuum, but rather by

$$
|\widetilde{g}\rangle=\widetilde{C}_{0+}|\widetilde{z}, 0\rangle\left|\chi_{\uparrow}\right\rangle-i \widetilde{C}_{0-}|\widetilde{z}, 1\rangle\left|\chi_{\downarrow}\right\rangle,
$$

which in addition to the two-mode squeezed vacuum, also includes the $\widetilde{n}_{r}=1 \mathrm{SU}(1,1)$ coherent state.

\section{Critical regime $\widetilde{\xi}=\xi$}

In this regime, the effective Hamiltonian can be directly obtained from Eq. (2)

$$
H^{c}:=H_{2 \mathrm{D}}^{\mathrm{free}}=c \sum_{j=1}^{2} \sigma_{j} p_{j}+\sigma_{z} m c^{2},
$$

which corresponds to the Dirac Hamiltonian of a twodimensional free fermion. In the critical regime $\tilde{\xi}=\xi$, the magnetic field coupling cancels the effect of the Dirac string coupling, and the fermion behaves as a free relativistic particles. The critical energy spectrum becomes

$$
E^{c}= \pm E_{\mathbf{p}}^{c}= \pm \sqrt{m^{2} c^{4}+\mathbf{p}^{2} c^{2}},
$$

where $\mathbf{p}=\left(p_{x}, p_{y}\right)$ stands for the two-dimensional fermion momentum. The corresponding eigenstates can be described as follows:

$$
\left| \pm E_{\mathbf{p}}^{c}\right\rangle=\sqrt{\frac{m c^{2} \pm E_{\mathbf{p}}^{c}}{ \pm 2 E_{\mathbf{p}}^{c}}}\left(\left|\chi_{\uparrow}\right\rangle+\frac{c\left(p_{x}+i p_{y}\right)}{m c^{2} \pm E_{\mathbf{p}}^{c}}\left|\chi_{\downarrow}\right\rangle\right)|\mathbf{p}\rangle,
$$

where $|\mathbf{p}\rangle:=\left|p_{x}, p_{y}\right\rangle$ are two-dimensional plane wave solutions. Note that these solutions describe the relativistic fermion at the critical point $\widetilde{\xi}=\xi$, but any small perturbation of the magnetic field or the Dirac string coupling will dramatically change the system properties. As we discuss below, this dramatic change shares many analogies with a quantum phase transition.

We have thus provided a complete solution of the relativistic Hamiltonian that describes the properties of a Dirac oscillator subjected to an additional constant magnetic field. A unitary transformation that connects the bichromatic full Hamiltonian with single-mode JC-like interactions has been described in Eqs. (13) and (23). With the aid of such transformation, one can obtain the exact energy spectrum for all

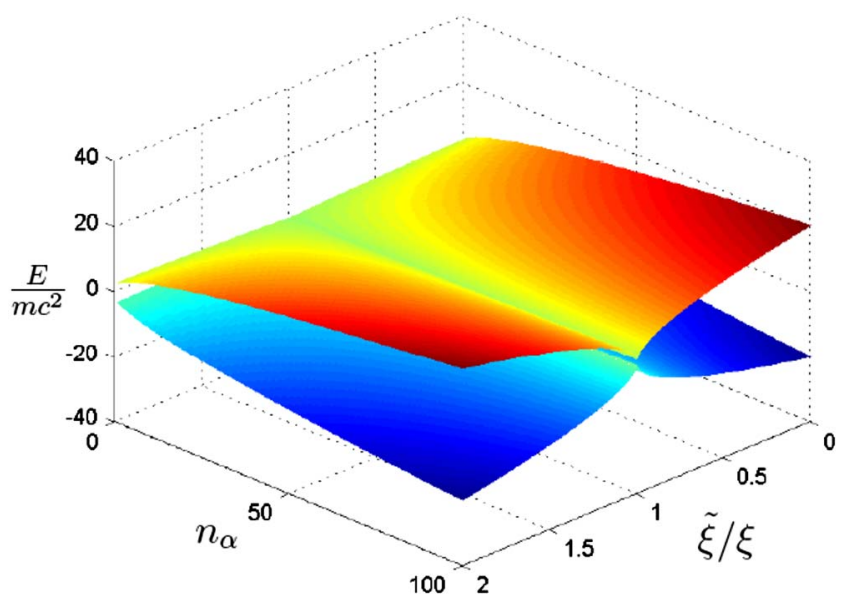

FIG. 2. (Color online) Fermionic energy spectrum as a function of the relative coupling strengths $\tilde{\xi} / \xi$ and the number of chiral phonons $n_{\alpha}\left(n_{\alpha}=n_{l}\right.$ if $\widetilde{\xi}<\xi$ and $n_{\alpha}=\tilde{n}_{r}$ if $\left.\widetilde{\xi}>\xi\right)$. The two energy sheets correspond to positive- and negative-energy solutions $\left| \pm E_{n_{l}}\right\rangle$ for $\tilde{\xi}<\xi$ and $\left| \pm \widetilde{E}_{\widetilde{n}_{r}}\right\rangle$ for $\tilde{\xi}>\xi$.

possible values of the coupling parameters $\xi, \widetilde{\xi}$, which responds to the analytical expressions in Eqs. (17) and (26). In this regard, one can study the transition between the weak $\tilde{\xi} / \xi \rightarrow 0$ and strong $\tilde{\xi} / \xi \rightarrow \infty$ coupling regimes which endows the relativistic system with a chiral symmetry change, as discussed in the previous section.

From a practical point of view, the magnetic field dependence of this relativistic system might be understood as an accessible method to prepare a certain relativistic state with specific properties. An adiabatic control of the applied magnetic field opens up the possibility of controlling a wide range of properties of this relativistic fermion, such as its chirality, squeezing, phonon statistics, and entanglement, which shall be discussed below.

\section{CHIRALITY QUANTUM PHASE TRANSITION}

In previous sections, we have described how the value of the relative coupling strength $\tilde{\xi} / \xi$ is responsible for the chirality of the system, which can be right or left handed by an appropriate tuning of the coupling strengths. This drastic modification can only be driven by means of quantum fluctuations, and therefore a quantum phase transitions occurs in this relativistic system. In this section we study several properties which are clear signatures of a quantum phase transition phenomenon, such as the vanishing of the gap at the critical point, the existence of an order parameter which takes on different values in the distinct chiral phases, the divergence of quantum fluctuations, and the maximization of entanglement across the critical point.

\section{A. Energy level crossing}

Here, we study the properties of the relativistic energy spectrum described in Eqs. (17) and (26), which are represented in Fig. 2. Here we represent the energies of different 


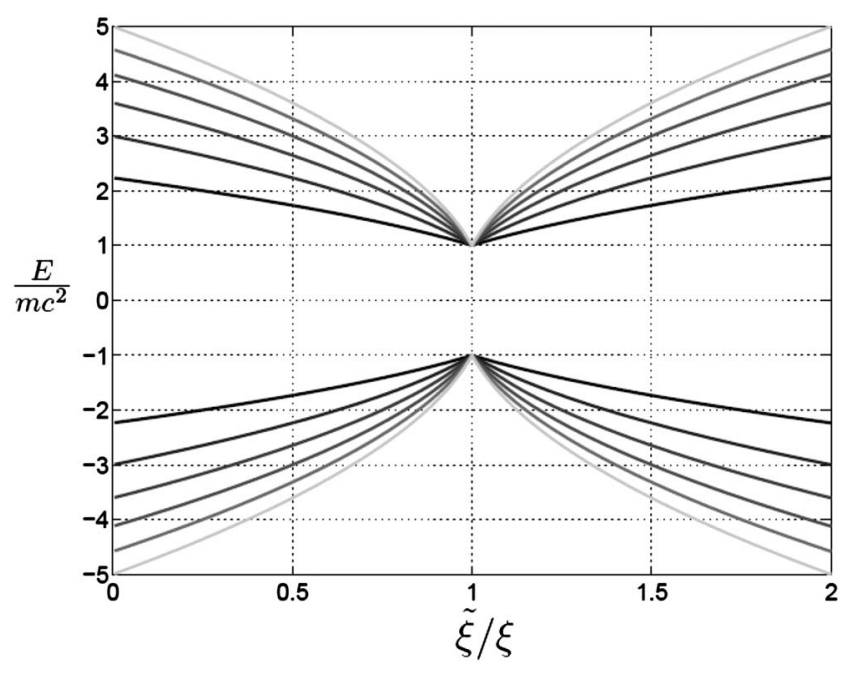

FIG. 3. Energy levels for the first excited eigenstates as a function of the relative coupling strengths $\tilde{\xi} / \xi$, showing the nonanalytic behavior at $(\widetilde{\xi} / \xi)_{c}=1$.

eigenstates $\left| \pm E_{n_{l}}\right\rangle,\left| \pm \widetilde{E}_{\widetilde{n}_{r}}\right\rangle$ with respect to the relative strength $\tilde{\xi} / \xi$.

We clearly observe two crucial signatures of a quantum phase transition (see also Fig. 3 for a two-dimensional sector of the above energy spectrum). The energy spectrum is nonanalytical for a critical coupling $(\tilde{\xi} / \xi)_{c}=1$. The system becomes gapless at this critical point $\Delta E \rightarrow 0$ when $\mid(\tilde{\xi} / \xi)$ $-(\tilde{\xi} / \xi)_{c} \mid \rightarrow 0$. Furthermore, we can obtain an analytical expression of the gap close to the critical point. In terms of the coupling strengths $g_{r}$ and $g_{l}$ in Eqs. (7) and (8), we obtain the following universal scaling law for the energy gap close to the critical point $\left(g_{r} / g_{l}\right)_{c}=(\tilde{\xi} / \xi)_{c}^{1 / 2}=1$ :

$$
\Delta E \sim m c^{2}\left|\frac{g_{r}}{g_{l}}-\left(\frac{g_{r}}{g_{l}}\right)_{c}\right|=: m c^{2}\left|\frac{g_{r}}{g_{l}}-\left(\frac{g_{r}}{g_{l}}\right)_{c}\right|^{z v},
$$

and we can readily identify the scaling exponents $z \nu=1$. The critical exponent $z$ is a dynamical exponent related to the decay of the system fluctuations with time, and in a Lorentz invariant model is expected to be $z=1$. Consequently, the remaining exponent, which characterizes the scaling of the correlation length close to the phase transition, becomes $\nu$ $=1$ in this relativistic model.

Finally, it is also important in quantum phase transitions to study the properties of the system ground state. In this case, the fermionic ground state in the left-handed regime is described by Eq. (22), while the ground state in the righthanded scenario becomes that of Eq. (31). The energy of such a ground state is nonanalytical at the critical point a follows from Fig. 4.

\section{B. Order parameter}

Another clear signature of quantum phase transitions is the appearance of an order parameter, a physical magnitude which acquires different values in the phases involved, and becomes indeterminate at the critical point. Therefore, an

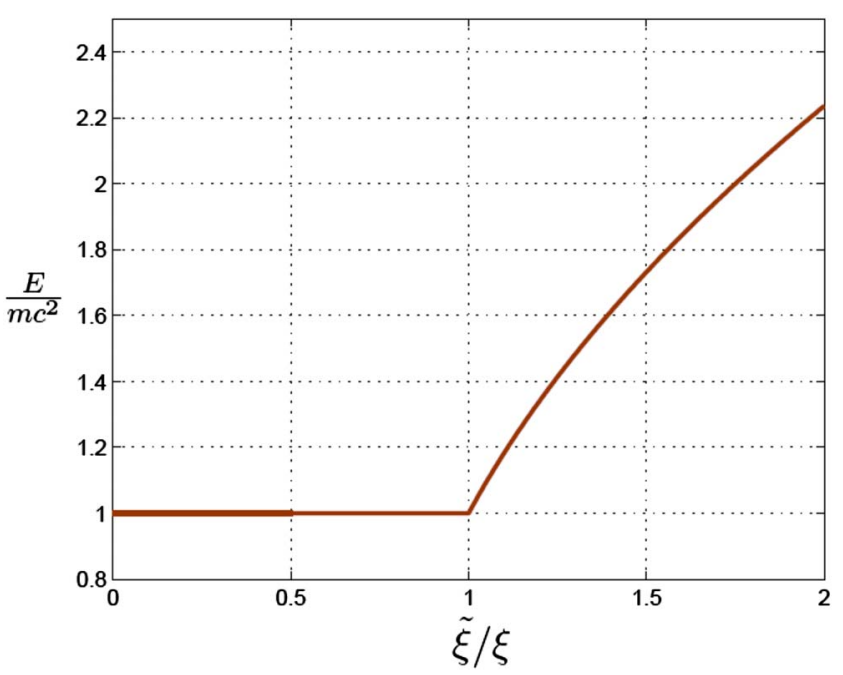

FIG. 4. (Color online) Ground-state energy as a function of the relative coupling $\tilde{\xi} / \xi$. As the magnetic filed is increased, the ground-state energy changes from $E_{g}=m c^{2} \rightarrow E_{\tilde{g}}=m c^{2} \sqrt{1+2 \zeta_{r}}$, which becomes nonanalytical at the critical point.

order parameter is able to witness the abrupt change in the properties of the system. In this relativistic scenario, the order parameter turns out to be the $z$ component of the orbital angular momentum $L_{z}=x p_{y}-y p_{x}$, an observable which is intimately related to the symmetry properties of the system, since $J_{z}=L_{z}+S_{z}$ is a conserved quantity. Note that this operator is also connected to the system chirality, since left-handed states fulfill $\left\langle L_{z}\right\rangle_{l} \leq 0$ whereas right-handed states fulfill $\left\langle L_{z}\right\rangle_{r} \geq 0$. In order to evaluate the angular momentum expectation value, it is useful to express the unitary transformations in Eqs. (19) and (28) in terms of canonical conjugate position and momentum operators

$$
\begin{aligned}
& U_{\widetilde{\alpha}}=e^{i(\alpha \tilde{\mu} / 4 \hbar)\left(x p_{x}+p_{x} x+y p_{y}+p_{y} y\right)} \quad \text { if } \quad \tilde{\xi}>\xi, \\
& U_{\widetilde{\alpha}}=e^{i(\tilde{\mu} \tilde{\mu} / 4 \hbar)\left(x p_{x}+p_{x} x+y p_{y}+p_{y} y\right)} \quad \text { if } \quad \tilde{\xi}>\xi .
\end{aligned}
$$

Using the transformations in Eqs. (36), one can obtain the expressions of the transformed position and momentum operators

$$
\begin{aligned}
& U_{\alpha} x_{i} U_{\alpha}^{\dagger}=e^{\alpha \tilde{\mu} / 2} x_{i}, \quad U_{\alpha} p_{i} U_{\alpha}^{\dagger}=e^{-(\alpha \tilde{\mu} / 2)} p_{i} \quad \text { if } \quad \tilde{\xi}<\xi, \\
& U_{\tilde{\alpha}} x_{i} U_{\tilde{\alpha}}^{\dagger}=e^{\tilde{\alpha} \tilde{\mu} / 2} x_{i}, \quad U_{\tilde{\alpha}} p_{i} U_{\tilde{\alpha}}^{\dagger}=e^{-(\tilde{\alpha} \tilde{\mu} / 2)} p_{i} \quad \text { if } \quad \tilde{\xi}>\xi,
\end{aligned}
$$

with $i=x, y$, which leads to the corresponding relations for the orbital angular momentum

$$
\begin{array}{lll}
U_{\alpha} L_{z} U_{\alpha}^{\dagger}=U_{\alpha}\left(x p_{y}-y p_{x}\right) U_{\alpha}^{\dagger}=L_{z} & \text { if } & \tilde{\xi}<\xi, \\
U_{\tilde{\alpha}} L_{z} U_{\tilde{\alpha}}^{\dagger}=U_{\tilde{\alpha}}\left(x p_{y}-y p_{x}\right) U_{\tilde{\alpha}}^{\dagger}=L_{z} & \text { if } & \tilde{\xi}>\xi .
\end{array}
$$

We observe from the expressions in Eqs. (38) that the $z$ component of the orbital angular momentum operator is not altered by the squeezing transformation. In the language of Lie 


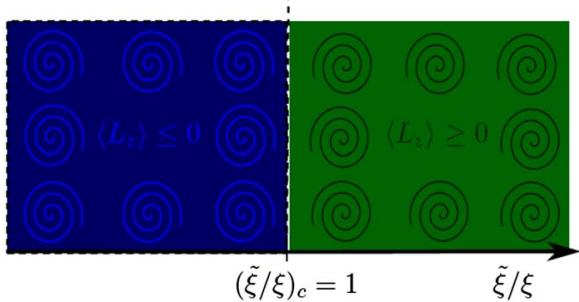

FIG. 5. (Color online) Mean value of the $z$ component of the orbital angular momentum (in units of $\hbar$ ) across the critical point. Note that, as the critical region is traversed, the sign of this order parameter changes and indicates that the phase transition has occurred.

algebras, this property is equivalent to $L_{z}$ being the Casimir operator associated with the underlying SU(1,1) algebra (see the Appendix). Therefore, we can easily obtain its expectation value in the eigenstates of Eqs. (18) and (27)

$$
\begin{aligned}
& \left\langle L_{z}\right\rangle_{\left| \pm E_{n_{l}}\right\rangle}=-\hbar\left(n_{l}+\frac{E_{n_{l}} \pm m c^{2}}{2 E_{n_{l}}}\right) \leq 0 \text { if } \tilde{\xi}<\xi, \\
& \left\langle L_{z}\right\rangle_{\mid \pm \tilde{E}_{\left.\tilde{n}_{r}\right\rangle}}=+\hbar\left(\tilde{n}_{r}+\frac{\tilde{E}_{\tilde{n}_{r}} \mp m c^{2}}{2 \widetilde{E}_{\tilde{n}_{r}}}\right) \geq 0 \text { if } \quad \tilde{\xi}>\xi,
\end{aligned}
$$

which show how the orbital angular momentum takes on negative values in the left-handed chiral phase, whereas it attains positive values in the right-handed chiral regime (see Fig. 5). Consequently, the orbital angular momentum plays the role of an order parameter which witnesses the quantum phase transition and macroscopically reveals such an effect by a change of its sign. Note that there is no apparent scaling law for the order parameter in the vicinity of the critical point. In this regard, the observable defined above is not a strict order parameter in the usual sense of quantum phase transitions, since it lacks the scaling property. Nonetheless, it still witnesses the phase transition and the abrupt change in the chirality, and we can thus consider it as an order parameter analog.

\section{Divergence of quantum fluctuations}

In the vicinity of a critical point, the gap of the system becomes negligible and excitations can be easily produced (see Fig. 3). Under such conditions, the system becomes highly fluctuating. As discussed previously, a quantum phase transition can only be driven by quantum fluctuations which lead to an abrupt change in the physical properties of the system when the critical region is crossed. In our case, it is possible to calculate analytically the divergences in the quantum fluctuations of the fermion position $\Delta x_{i}=\sqrt{\left\langle x_{i}^{2}\right\rangle-\left\langle x_{i}\right\rangle^{2}}$.

We shall not only focus in the ground-state fluctuations in Eqs. (22) and (31), but we shall investigate the quantum fluctuations of the whole energy eigenstates in Eqs. (21) and (30). Using the transformations described in Eq. (37), we obtain

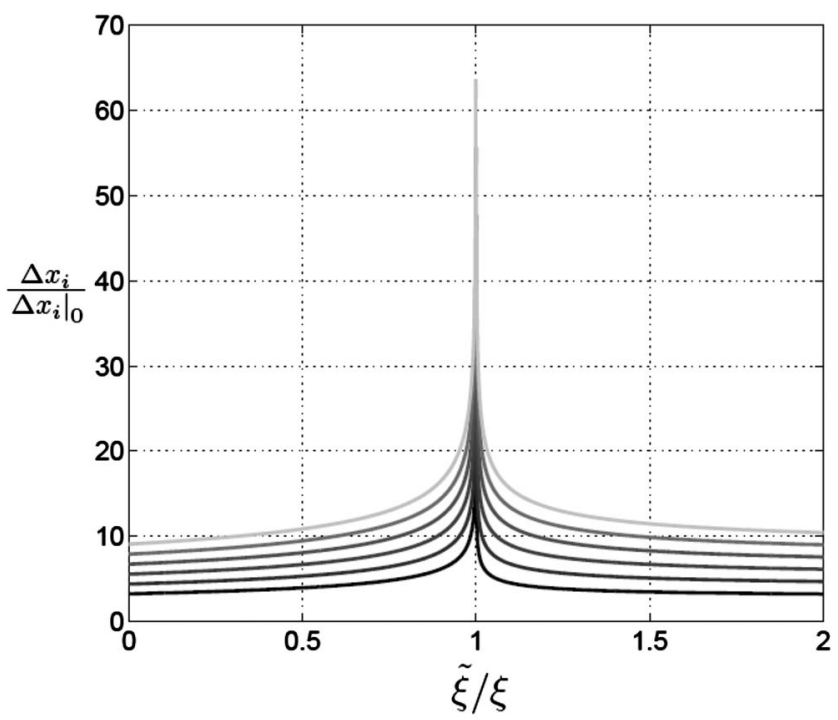

FIG. 6. Quantum fluctuations in the fermion position $\Delta x_{i}$ (in units of vacuum fluctuations $\left.\Delta x_{i}\right|_{0}$ ) for the first excited positiveenergy eigenstates $\left|+E_{n_{l}}\right\rangle,\left|+\widetilde{E}_{\tilde{n}_{r}}\right\rangle$ as a function of the relative coupling strengths $\widetilde{\xi} / \xi$. We note that the negative-energy eigenstates display similar fluctuations in position.

$$
\begin{aligned}
& \left.\Delta x_{i}\right|_{\left. \pm \pm E_{n_{l}}\right\rangle}=\left.\Delta x_{i}\right|_{\mathrm{vac}} \sqrt{\eta_{ \pm}} e^{+(\alpha \tilde{\mu} / 2)} \quad \text { if } \quad \tilde{\xi}<\xi, \\
& \left.\Delta x_{i}\right|_{\left. \pm \pm \tilde{E}_{\tilde{n}_{r}}\right\rangle}=\left.\tilde{\Delta} x_{i}\right|_{\mathrm{vac}} \sqrt{\widetilde{\eta}_{\mp}} e^{+(\tilde{\alpha} \tilde{\mu} / 2)} \quad \text { if } \quad \widetilde{\xi}>\xi,
\end{aligned}
$$

where $i=x, y,\left.\Delta x_{i}\right|_{\mathrm{vac}}:=\Delta / \sqrt{2}$ stand for the fluctuations of the vacuum in modes $a_{r}, a_{l}$, whereas $\left.\widetilde{\Delta} x_{i}\right|_{\text {vac }}:=\widetilde{\Delta} / \sqrt{2}$ are the vacuum fluctuations in modes $\tilde{a}_{r}, \tilde{a}_{l}$. We have also introduced $\eta_{ \pm}:=n_{l}+3 / 2 \pm m c^{2} / 2 E_{n_{l}}$ and $\tilde{\eta}_{ \pm}:=\tilde{n}_{r}+3 / 2 \pm m c^{2} / 2 \widetilde{E}_{\widetilde{n}_{r}}$, which depend on the number of quanta. We have represented in Fig. 6 the fluctuations in the fermion position for different eigenstates as the relative coupling $\widetilde{\xi} / \xi$ is varied. We observe how these fluctuations diverge at the critical point $(\tilde{\xi} / \xi)_{c}=1$ for all the eigenstates, representing yet another signature of a quantum phase transition.

Nonetheless, we have remarked in Eqs. (19) and (28) that the unitary transformation involved corresponds to a squeezing transformation in the language of quantum optics. As a consequence, a squeezing of certain fluctuations must also become apparent for a certain observable. This is the case of the fermionic momentum, whose fluctuations $\Delta p_{i}$ $=\sqrt{\left\langle p_{i}^{2}\right\rangle-\left\langle p_{i}\right\rangle^{2}}$ for different eigenstates are the following:

$$
\begin{aligned}
& \left.\Delta p_{i}\right|_{\left. \pm E_{n_{l}}\right\rangle}=\left.\Delta p_{i}\right|_{\mathrm{vac}} \sqrt{\eta_{n_{l}, \pm}} e^{-(\alpha \tilde{\mu} / 2)} \text { if } \quad \widetilde{\xi}<\xi, \\
& \left.\Delta p_{i}\right|_{\left| \pm \tilde{E}_{\tilde{n}_{r}}\right\rangle}=\left.\tilde{\Delta} p_{i}\right|_{\mathrm{vac}} \sqrt{\eta_{\tilde{n}_{r},}, e^{-(\tilde{\alpha} \tilde{\mu} / 2)} \quad \text { if } \quad \tilde{\xi}>\xi}
\end{aligned}
$$

where $\left.\Delta p_{i}\right|_{\mathrm{vac}}=\hbar / \Delta \sqrt{2}$ and $\left.\widetilde{\Delta} p_{i}\right|_{\mathrm{vac}}=\hbar / \widetilde{\Delta} \sqrt{2}$ stand for the vacuum fluctuations in each regime. Remarkably, the fluctuations in the momentum vanish as the system approaches the critical point (see Fig. 7). Therefore, the squeezing occurs in the relativistic momentum. 


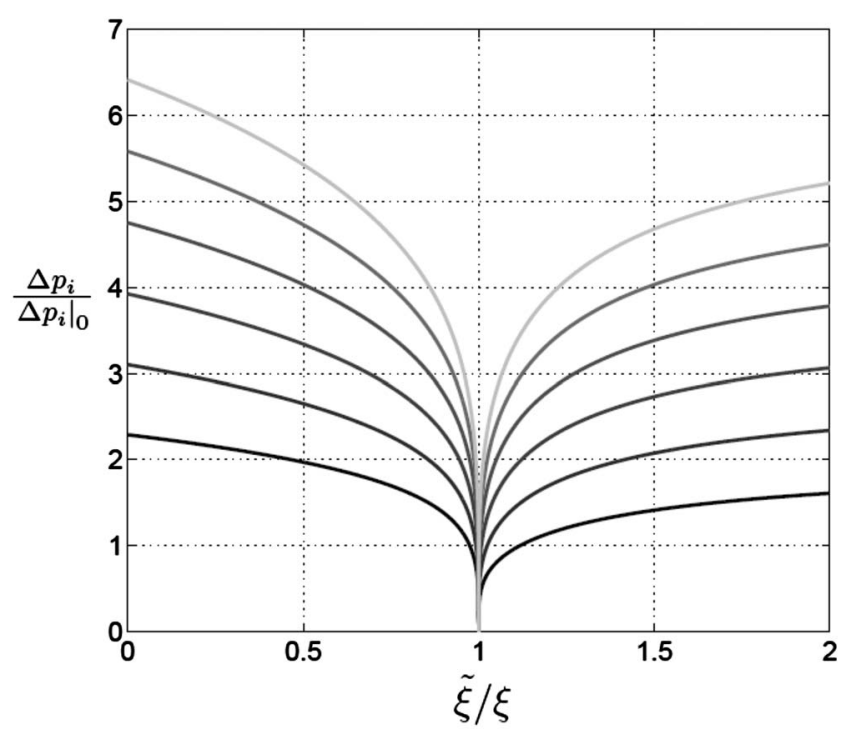

FIG. 7. Quantum fluctuations in the fermion momentum $\Delta p_{i}$ (in units of vacuum fluctuations $\left.\Delta p_{i}\right|_{0}$ ) for the first excited positiveenergy eigenstates $\left|+E_{n_{l}}\right\rangle,\left|+\widetilde{E}_{\widetilde{n}_{r}}\right\rangle$ as a function of the relative coupling strengths $\widetilde{\xi} / \xi$. We note that the negative-energy eigenstates display similar fluctuations in momentum.

With these observations, we have a full description of the system for any value of the relative coupling. Left-handed regime $\tilde{\xi}<\xi$ : The relativistic eigenstates are described by an AJC-like doublet of SU $(1,1)$ coherent states entangled with Pauli spinors (21), which are rotating clockwise [see Eq. (39)]. Critical regime $\tilde{\xi}=\xi$ : The critical properties are described by those of a relativistic free fermion. Right-handed regime $\widetilde{\xi}>\xi$ : The relativistic eigenstates are described by an JC-like doublet of SU $(1,1)$ coherent states entangled with Pauli spinors (30), which are rotating counterclockwise [see Eq. (39)].

\section{Phonon statistical properties}

In this section we study the statistical properties of the chiral phonon distribution. The statistical properties of a bosonic ensemble can be classified according to its quantum fluctuations. Poissonian statistics: In this case, the phonon number distribution is a Poissonian random variable. Quantum fluctuations in the number of bosons fulfill $\Delta n=\sqrt{\langle n\rangle}$, where $n=a^{\dagger} a$ is the bosonic number operator and $\Delta n$ $=\sqrt{\left\langle n^{2}\right\rangle-\langle n\rangle^{2}}$. Super-Poissonian statistics: In this regime, the quantum fluctuations of the bosonic ensemble are bigger with respect to the Poissonian distribution $\Delta n>\sqrt{\langle n\rangle}$, and therefore the phonons are said to be noisier. Sub-Poissonian statistics: The quantum nature of a bosonic ensemble allows a further possibility, which is evidence of nonclassical behavior, that of sub-Poissonian statistics. Under these circumstances, the statistical fluctuations become lower than those of a Poissonian distribution $\Delta n<\sqrt{\langle n\rangle}$ and consequently the bosons are said to be quieter quantum entities.

In this section we show how the relativistic phonon distribution attains different statistical properties depending on the relative coupling parameter $\tilde{\xi} / \xi$. In particular, we show how the quantum noise of the chiral phonon distribution changes from sub-Poissonian to super-Poissonian as the critical point is crossed. In order to quantify the Poissonian character of the chiral boson ensemble, we shall make use of the chiral Mandel $Q$ parameters, defined as follows:

$$
Q_{r}=\frac{\left(\Delta n_{r}\right)^{2}}{\left\langle n_{r}\right\rangle}-1, \quad Q_{l}=\frac{\left(\Delta n_{l}\right)^{2}}{\left\langle n_{l}\right\rangle}-1 .
$$

A positive sign in such parameters reveals the superPoissonian nature of the bosonic ensemble, whereas a negative sign shows the sub-Poissonian bosonic statistics which stress the nonclassical nature of the ensemble. The corresponding fluctuations in the system eigenstates (21) and (30), can be calculated using the expressions in Eqs. (A6) and (A7) in the Appendix. In the left-handed regime $\widetilde{\xi}<\xi$, we have found

$$
\begin{gathered}
\left\langle n_{r}\right\rangle_{\left| \pm E_{n_{l}}\right\rangle}=\eta_{ \pm} \sinh ^{2}|z|, \\
\left\langle n_{l}\right\rangle_{\left| \pm E_{n_{l}}\right\rangle}=\eta_{ \pm} \cosh ^{2}|z|-1, \\
\left.\Delta n_{r}\right|_{\left| \pm E_{n_{l}}\right\rangle} ^{2}=\eta_{ \pm} \sinh ^{2}|z| \cosh ^{2}|z|+\kappa \sinh ^{4}|z|, \\
\left.\Delta n_{l}\right|_{\left| \pm E_{n_{l}}\right\rangle} ^{2}=\eta_{ \pm} \sinh ^{2}|z| \cosh ^{2}|z|+\kappa \cosh ^{4}|z|,
\end{gathered}
$$

where the parameters $\eta_{ \pm}$have been previously introduced and $\kappa:=\frac{1}{4}\left(1-m^{2} c^{4} / E_{n_{l}}^{\prime 2}\right)$. Analogously, in the right-handed case $\tilde{\xi}>\xi$

$$
\begin{gathered}
\left\langle\tilde{n}_{r}\right\rangle_{\left| \pm \tilde{E}_{\tilde{n}_{r}}\right\rangle}=\tilde{\eta}_{\mp} \cosh ^{2}|\tilde{z}|-1, \\
\left\langle\tilde{n}_{l}\right\rangle_{\left| \pm \tilde{E}_{\tilde{n}_{r}}\right\rangle}=\tilde{\eta}_{\mp} \sinh ^{2}|\tilde{z}|,
\end{gathered}
$$

$$
\begin{aligned}
& \left.\Delta \tilde{n}_{r}\right|_{\left| \pm \tilde{E}_{\tilde{n}_{r}}\right\rangle} ^{2}=\tilde{\eta}_{\mp} \sinh ^{2}|\tilde{z}| \cosh ^{2}|\tilde{z}|+\widetilde{\kappa} \cosh ^{4}|\tilde{z}|, \\
& \left.\Delta \tilde{n}_{l}\right|_{\left| \pm \tilde{E}_{\tilde{n}_{r}}\right\rangle} ^{2}=\tilde{\eta}_{\mp} \sinh ^{2}|\tilde{z}| \cosh ^{2}|\tilde{z}|+\widetilde{\kappa} \sinh ^{4}|\tilde{z}|,
\end{aligned}
$$

where $\tilde{\eta}_{ \pm}$have already been introduced, and the parameter $\tilde{\kappa}:=\frac{1}{4}\left(1-m^{2} c^{4} / E_{\tilde{n}_{r}}^{\prime 2}\right)$. These expressions allow us to study in detail the statistical nature of the chiral phonon ensemble by means of the Mandel parameters in Eq. (42), which have been represented in Figs. 8 and 9. In this figures we notice the following. Left-handed regime $\tilde{\xi}<\xi$ : In this limit, the chiral Mandel parameters become $Q_{l}<0 \Rightarrow$ sub-Poissonian statistics of quieter left-handed phonons $Q_{r}>0$ $\Rightarrow$ super-Poissonian statistics of noisier right-handed phonons. Right-handed regime $\widetilde{\xi}>\xi$ : In this limit, the chiral Mandel parameters become $Q_{l}>0 \Rightarrow$ super-Poissonian statistics of noisier left-handed phonons, $Q_{r}<0 \Rightarrow$ sub-Poissonian statistics of quieter right-handed phonons.

Therefore, we observe how the statistical nature of the relativistic phonons is completely controlled by the relative coupling strength. Remarkably enough, the level of quantum 


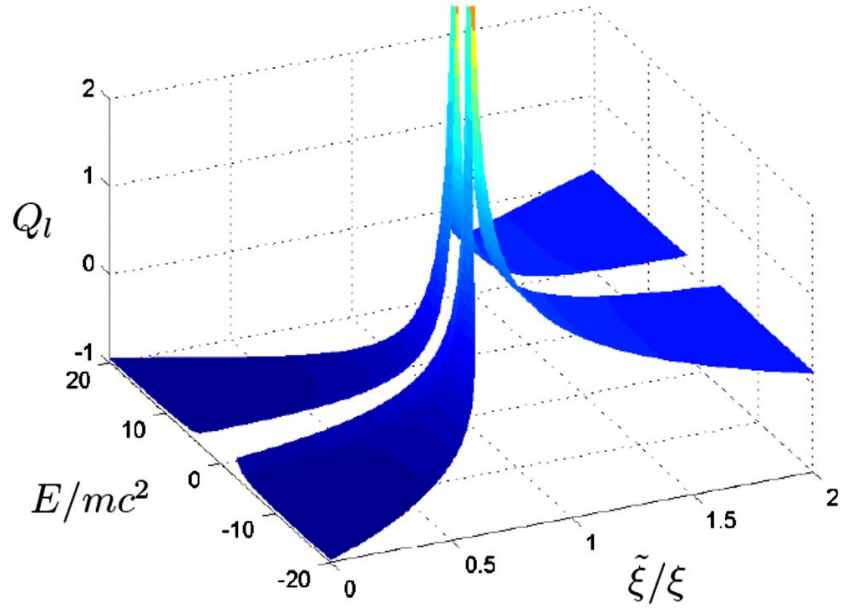

FIG. 8. (Color online) Left-handed Mandel parameter $Q_{l}$ for the energy eigenstates $\left\{\left| \pm E_{n_{l}}\right\rangle,\left| \pm \widetilde{E}_{\widetilde{n}_{r}}\right\rangle\right\}$ as a function of the relative coupling strengths $\tilde{\xi} / \xi$. Note that as the magnetic field is raised, the ensemble of left-handed phonons changes from sub- to superPoissonian statistics.

noise in the chiral ensembles can be controlled by means of external parameters. Furthermore, a notorious transition between sub- and super-Poissonian statistics occurs as the system crosses the critical region. This fact shows how a quantum phase transition can also be intrinsically related to the statistical nature of the system. Note also that close to the critical point $Q_{l}, Q_{r} \gg 1$, and both ensembles become superPoissonian indicating how quantum fluctuations diverge at the critical point of a quantum phase transition.

\section{E. Entanglement at the critical point}

In previous sections, we have seen how several signatures of a quantum phase transition occur in this relativistic sys-

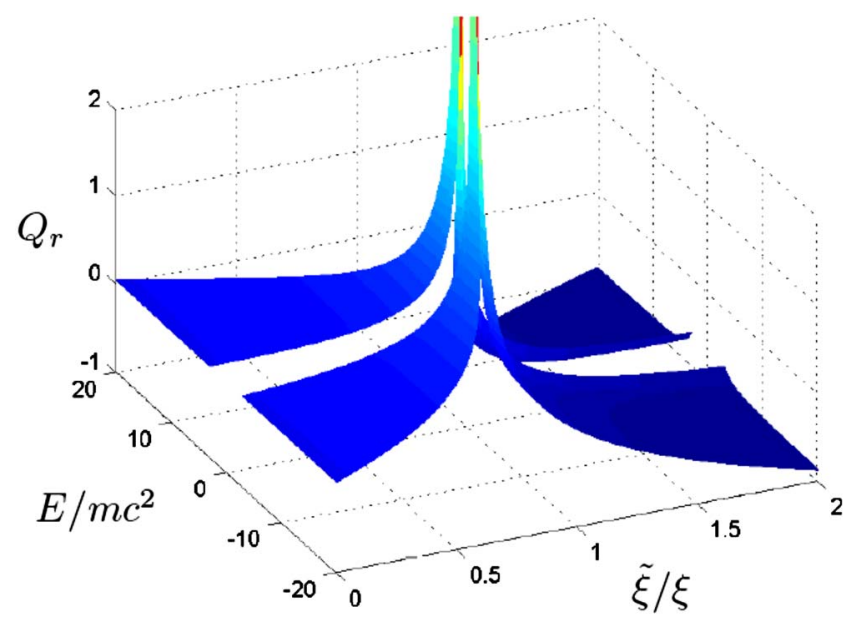

FIG. 9. (Color online) Right-handed Mandel parameter $Q_{r}$ for the energy eigenstates $\left\{\left| \pm E_{n_{l}}\right\rangle,\left| \pm \widetilde{E}_{\widetilde{n}_{r}}\right\rangle\right\}$ as a function of the relative coupling strengths $\widetilde{\xi} / \xi$. Note that as the magnetic field is raised, the ensemble of right-handed phonons changes from super- to subPoissonian statistics.

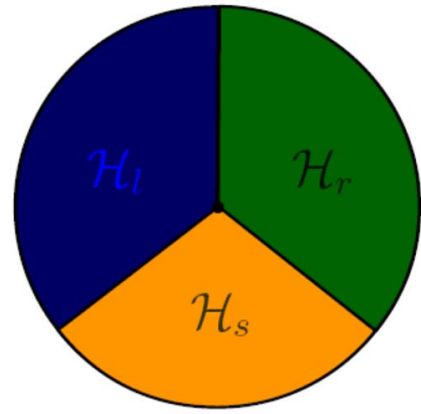

FIG. 10. (Color online) The relativistic degrees of freedom can be described as a tripartite system with a Hilbert space $\mathcal{H}=\mathcal{H}_{r}$ $\otimes \mathcal{H}_{l} \otimes \mathcal{H}_{s}$ that includes continuous-variables corresponding to the chiral degrees of freedom, and discrete variables related to the spinorial degree of freedom.

tem. We have described in detail the closure of the energy gap at the critical point, the existence of an order parameter which turns out to be the orbital angular momentum, and the divergence of quantum fluctuations as one explores the critical region. An additional feature in quantum phase transitions is the divergence of quantum correlations as the system crosses the critical region [20,21]. Correlations of a quantum nature, known as entanglement, lie at the heart of quantum mechanics and are responsible for nonlocal phenomena in the quantum regime. Furthermore, they are of utmost relevance in the fields of quantum information and computation, where they constitute a resource for information processing tasks [22-24]. In the particular case of fermions, entanglement has been previously studied for relativistic field theory [25,26] (see Ref. [27] for a recent review). In this section we provide a description of fermionic entanglement across a quantum phase transition, that of a relativistic Dirac oscillator subjected to a magnetic field.

In our case, the underlying Hilbert space can be described as a tripartite system composed of continuous-variables associated to the chiral degrees of freedom, and discrete variables related to the spin degree of freedom (see Fig. 10). To quantify quantum correlations in this hybrid system we make use of the machinery of discrete-variable [28] and continuous-variable [29] entanglement measures. In particular, we obtain the von Neumann entropy of the reduced density matrices over all possible bipartitions

$$
\begin{gathered}
\rho_{l}:=\operatorname{Tr}_{s}\left(\operatorname{Tr}_{r}|\psi\rangle\langle\psi|\right), \quad \rho_{r}:=\operatorname{Tr}_{s}\left(\operatorname{Tr}_{l}|\psi\rangle\langle\psi|\right), \\
\rho_{s}:=\operatorname{Tr}_{l}\left(\operatorname{Tr}_{r}|\psi\rangle\langle\psi|\right),
\end{gathered}
$$

where the subindexes $l, r, s$ stand for the left-handed, righthanded, and spinorial degrees of freedom, $|\psi\rangle$ is a particular pure state, and the von Neumann entropy is defined as $S(\rho):=-\operatorname{Tr}(\rho \log \rho)$. Such magnitude, when calculated over reduced density matrices, quantifies the amount of entanglement between two parties. In our case, it quantifies the amount of entanglement between different relativistic degrees of freedom.

Left-handed regime $\tilde{\xi}<\xi$. The ground state of the relativistic spin-1/2 oscillator in Eq. (22) is described by means of 


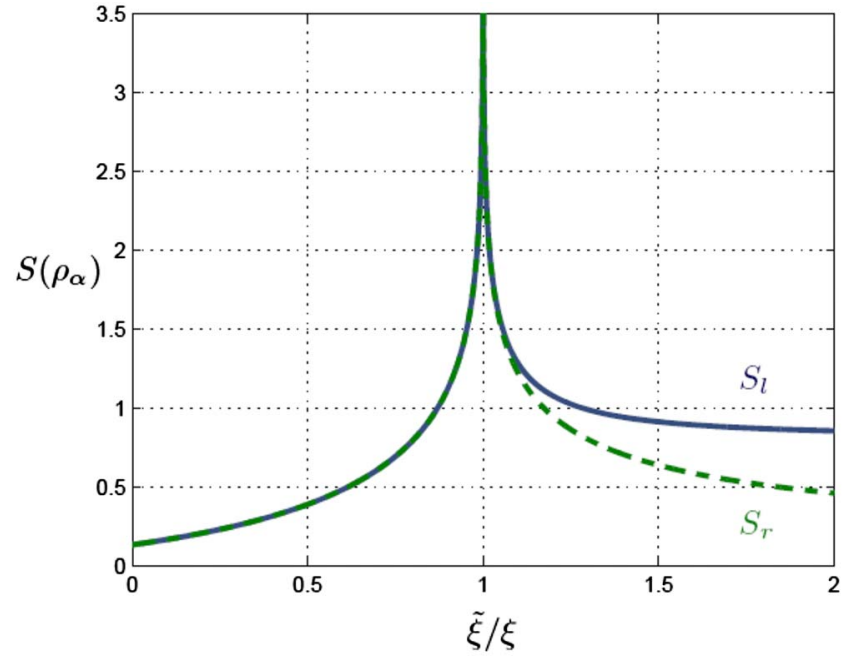

FIG. 11. (Color online) Von Neumann entropy of the singlemode reduced states $\rho_{\alpha}$, with $\alpha=l, r$ indexing the mode chirality, as a function of the coupling strength ratio $\tilde{\xi} / \xi$. One directly observes that the entanglement of the continuous-variable degrees of freedom with the rest of the system diverges as one approaches the critical region.

a spin-up two-mode squeezed vacuum state, with the following reduced density matrices:

$$
\begin{gathered}
\rho_{l}=\frac{1}{\cosh ^{2}|z|} \sum_{n_{l}=0}^{\infty} \tanh ^{2 n_{l}}|z|\left|n_{l}\right\rangle\left\langle n_{l}\right|, \\
\rho_{r}=\frac{1}{\cosh ^{2}|z|} \sum_{n_{r}=0}^{\infty} \tanh ^{2 n_{r}}|z|\left|n_{r}\right\rangle\left\langle n_{r}\right|, \\
\rho_{s}=\left|\chi_{\uparrow}\right\rangle\left\langle\chi_{\uparrow}\right| .
\end{gathered}
$$

The reduced chiral degrees of freedom are described by means of a mixed thermal state with an effective temperature $T_{\text {eff }}:=\hbar \omega / 2 k_{B} \log (\operatorname{coth}|z|)$, where $k_{B}$ is the Boltzmann constant. Remarkably, the entropy of a thermal state can be given in terms of its mean number of quanta $\langle n\rangle[30,31]$, and we obtain $S_{l}:=S\left(\rho_{l}\right)=\sinh ^{2}|z| \log \left(1+\operatorname{cosech}^{2}|z|\right)$ $+\log \left(\cosh ^{2}|z|\right)=S\left(\rho_{r}\right)=: S_{r}$. There exists thus a certain amount of chiral entanglement, which depends on the relative coupling strength and diverges as one approaches the critical point $(\tilde{\xi} / \xi)_{c}=1$ (see Fig. 11). Conversely, the reduced spin density matrix is in a spin-up pure state with vanishing entropy $S_{s}:=S\left(\rho_{s}\right)=0$ (see Fig. 12). This remarks the fact that there is no spin-orbit entanglement between the discreteand continuous-degrees of freedom, which is in accordance to the ground state structure in Eq. (22), which is biseparable with respect to the spin-orbit bipartition.

Right-handed regime $\tilde{\xi}>\xi$. In this limit, the relativistic ground state in Eq. (31) can no longer be described by a spin-up squeezed vacuum state, but rather by means of a SU $(1,1)$ doublet, where chiral coherent states are entangled with spin states. The reduced density matrices become

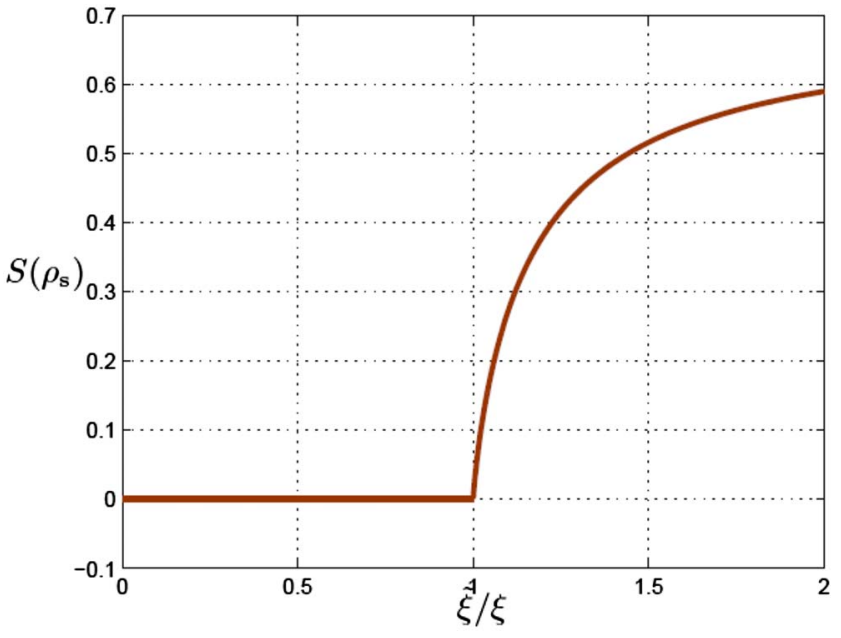

FIG. 12. (Color online) Von Neumann entropy of the spinorial reduced state $\rho_{s}$ as a function of the coupling strength ratio $\tilde{\xi} / \xi$. We observe that the entanglement of the spin degree of freedom to the rest of the system increases with the magnetic field.

$$
\begin{gathered}
\rho_{l}=\frac{1}{\cosh ^{2}|\tilde{z}|} \sum_{\tilde{n}_{l}=0}^{\infty} \tanh ^{2 \tilde{n}_{l} \mid}|\tilde{z}|\left(\gamma_{+}+\frac{\gamma_{-} \tilde{n}_{l}}{\sinh ^{2}|\tilde{z}|}\right)\left|\tilde{n}_{l}\right\rangle\left\langle\tilde{n}_{l}\right|, \\
\rho_{r}=\frac{1}{\cosh ^{2}|\tilde{z}|_{\tilde{n}_{r}=0}^{\infty}} \sum_{\tanh ^{2 \tilde{n}_{r}}|\tilde{z}|}^{\infty}\left(\gamma_{+}+\frac{\gamma_{-}\left(\tilde{n}_{r}+1\right)}{\cosh ^{2}|\tilde{z}|}\right)\left|\tilde{n}_{r}\right\rangle\left\langle\tilde{n}_{r}\right|, \\
\rho_{s}=\gamma_{+}\left|\chi_{\uparrow}\right\rangle\left\langle\chi_{\uparrow}\left|+\gamma_{-}\right| \chi_{\downarrow}\right\rangle\left\langle\chi_{\downarrow}\right|,
\end{gathered}
$$

where $\gamma_{ \pm}:=\left(\sqrt{1+2 \zeta_{r}} \pm 1\right) / 2 \sqrt{1+2 \zeta_{r}}$. In this regime, the reduced chiral degrees of freedom cannot be described by means of effective thermal states, nor can the reduced spin degrees of freedom be identified with a pure state. Nonetheless, the density matrices in Eqs. (47) are already in diagonal form, and the von Neumann entropy can be expressed as follows:

$$
\begin{gathered}
S_{l}=-\sum_{\widetilde{n}_{l}=0}^{\infty} \Theta_{\widetilde{n}_{l}}^{l} \log \Theta_{\tilde{n}_{l}}^{l}, \\
S_{r}=-\sum_{\widetilde{n}_{r}=0}^{\infty} \Theta_{\widetilde{n}_{r}}^{r} \log \Theta_{\tilde{n}_{r}}^{r} \\
S_{S}=-\frac{1}{2}\left[\log \left(\frac{\zeta_{r}}{2\left(1+\zeta_{r}\right)}\right)+\frac{1}{\sqrt{1+2 \zeta_{r}}} \log \left(\frac{\sqrt{1+2 \zeta_{r}}+1}{\sqrt{1+2 \zeta_{r}}-1}\right)\right],
\end{gathered}
$$

with the parameters $\Theta_{\widetilde{n}_{l}}^{l}:=\frac{\tanh ^{2} \tilde{n}_{n}|\tilde{z}|}{\cosh ^{2}|\tilde{z}|}\left(\gamma_{+}+\frac{\gamma_{-} \widetilde{n}_{l}}{\sinh ^{2}|\tilde{z}|}\right)$ and $\Theta_{\widetilde{n}_{r}}^{r}$ $:=\frac{\tanh ^{2 \tilde{r}_{r} \mid z}}{\cosh ^{2}|z|}\left(\gamma_{+}+\frac{\gamma_{-}\left(\widetilde{n}_{r}+1\right)}{\cosh ^{2}|z|}\right)$. These magnitudes, which represent entanglement between all possible bipartitions of the system have been represented in Figs. 11 and 12. We observe certain peculiarities with respect to the left-handed regime, such as a finite spinorial entanglement which tends to its maximal value $S_{s}^{\max }=\log 2$ as the magnetic field is raised $\widetilde{\xi} / \xi \rightarrow \infty$. We 
also observe that the continuous-variable entanglement is no longer equal for opposed chiral modes, which is a consequence of a finite orbital angular momentum in the ground state. In addition, one can see from Eq. (31) that the relativistic ground state presents genuine tripartite entanglement, where all the degrees of freedom are correlated among each other.

\section{CONCLUSIONS}

In this work we have studied the properties of a relativistic spin-1/2 Dirac oscillator subjected to a constant magnetic field. The relativistic Hamiltonian can be mapped onto a couple of Jaynes-Cummings and anti-Jaynes-Cummings terms, which describe the interaction between the relativistic spinor and bosons that carry an intrinsic chirality. These models, which are of paramount importance in quantum optics, become useful in this relativistic scenario, and allow an insightful description of interplay between opposed chirality interactions. The study of this relativistic system in the limits of a weak and strong magnetic field points toward the existence of two different phases, each described by an opposed chirality. In the intermediate regime, an intriguing trade-off between left- and right-handed interactions leads to the appearance of a quantum phase transition, which we have described in full detail.

The remarkable possibility to find the exact solution of this relativistic system has allowed us to describe analytically several properties of this phase transition. We have discussed the nonanalytic nature of the energy spectrum at the critical point, and the sudden extinction of the energy gap, which are archetypical properties of quantum phase transitions. We have also proved that there is an order parameter, the $z$ component of the orbital angular momentum, that witnesses the chiral phase change. In the critical region, we have shown that the quantum fluctuations in the fermion position diverge, which is a typical sign of quantum phase transition. Conversely, the quantum fluctuations in momentum vanish at the critical point, since a particular squeezing of this canonical variable occurs due to the interplay of the interactions. We have discussed the bosonic ensemble statistics, and their behavior across the phase transition. In this regard, we have found that super- or sub-Poissonian nature of such ensemble can be controlled at will by an appropriate modification of the magnetic field strength.

Entanglement properties between the various degrees of freedom of this relativistic system has also been accomplished. This hybrid system consists of a couple of continuous-variable degrees of freedom associated to the chiral modes, and a discrete-variable associated to the spin, therefore constituting a tripartite system. We have shown that an entanglement measure, the von Neumann entropy of the reduced states, over all possible bipartitions can be obtained for the system ground state. At the critical point, the quantum correlations of the chiral degrees of freedom diverge, which can be also considered as a sign of quantum phase transitions.

Finally, let us also comment on the possibility of realizing the Dirac Hamiltonian of Eq. (2) in an ion trap experiment.
As proposed in Ref. [10], it is possible to obtain an effective Hamiltonian equivalent to the bare $2+1$ Dirac oscillator. The same techniques would suffice to include additional terms that describe the magnetic field coupling in Eq. (2). For example, this can be achieved by a suitable combination of two stationary waves tuned above and below the resonance frequency [32] (note that the tilded annihilation operators do not represent independent modes with respect the untilded operators, as pointed out in Ref. [17]). Therefore, once the $2+1$ Dirac oscillator has been realized with an ion trap tabletop, it seems straightforward to simulate the chirality quantum phase transition described in this paper. An alternative approach, which would provide us with states with a remarkable chirality, squeezing, phonon statistics, and entanglement, is to perform the couple of squeezing transformations described in Eqs. (36) to a trapped ion subjected to first blueor red-sideband excitations in Eqs. (16) and (25). The squeezing transformations can be achieved following different procedures, such as a sudden variation of the trapping frequencies $[33,34]$.

\section{ACKNOWLEDGMENTS}

We acknowledge financial support from Spanish MEC Project No. FIS2006-04885, Project No. CAM-UCM/910758 (A.B. and M.A.M.D.) and UCM Project No. PR1-A/0715378 (A.L.). Additionally, we acknowledge support from a FPU MEC grant (A.B.), and ESF Science Programme No. INSTANS 2005-2010 (M.A.M.D.).

\section{APPENDIX: SU(1,1) COHERENT STATES}

In this appendix we review the properties of two-mode SU $(1,1)$ coherent states. Such states arose from a group theoretical approach to the generalization of the usual Glaubler coherent states [35], which can be constructed for an arbitrary Lie group [36]. In this case, we shall restrict our attention to the $\mathrm{SU}(1,1)$ group, whose generators $\left\{K_{0}, K_{+}, K_{-}\right\}$ have the following Lie algebra:

$$
\left[K_{0}, K_{ \pm}\right]= \pm K_{ \pm}, \quad\left[K_{-}, K_{+}\right]=2 K_{0} .
$$

The Schwinger representation of algebra (A1) consists of a pair of commuting bosonic harmonic oscillator operators $a_{r}, a_{l}$ related to the group generators as follows [37,38]:

$$
K_{0}=\frac{1}{2}\left(a_{r}^{\dagger} a_{r}+a_{l}^{\dagger} a_{l}+1\right), \quad K_{+}=a_{r}^{\dagger} a_{l}^{\dagger}=\left(K_{-}\right)^{\dagger} .
$$

This two-mode representation is very useful, since one can use the disentangling theorem [36] to calculate the $\operatorname{SU}(1,1)$ coherent states $\left|z, n_{l}\right\rangle=U_{\alpha}^{\dagger}\left|n_{l}\right\rangle|\mathrm{vac}\rangle_{r}$ in Eq. (20) in the usual Fock basis $\left|n_{r}, n_{l}\right\rangle$

$$
U_{\alpha}^{\dagger}=e^{z\left(K_{+}-K_{-}\right)}=e^{\tanh z K_{+}} e^{\log \left(\cosh ^{-2} z\right) K_{0} / 2} e^{-\tanh z K_{-}} .
$$

Such factorization of the exponential (A3) allows the following expression for the $\mathrm{SU}(1,1)$ coherent states: 


$$
\left|z, n_{l}\right\rangle=\mathcal{N}_{n_{l}} \sum_{m=0}^{\infty} \sqrt{\frac{\left(m+n_{l}\right) !}{n_{l} ! m !}}(-1)^{m} \tanh ^{m}|z|\left|m+n_{l}, m\right\rangle,
$$

which has been used in Eq. (20) of the left-handed regime $\xi>\widetilde{\xi}$. Analogously, one can obtain the corresponding $\mathrm{SU}(1,1)$ coherent states in the right-handed regime, using a similar disentangling theorem

$$
\left|\widetilde{z}, \tilde{n}_{r}\right\rangle=\mathcal{N}_{\tilde{n}_{r}} \sum_{\tilde{m}=0}^{\infty} \sqrt{\frac{\left(\tilde{m}+\tilde{n}_{r}\right) !}{\tilde{n}_{r} ! \tilde{m} !}}(-1)^{\tilde{m}} \tanh ^{\tilde{m}}|\tilde{z}|\left|\tilde{m}+\tilde{n}_{r}, \tilde{m}\right\rangle,
$$

which was used in Eq. (29).

In the Lie algebraic language, the Casimir operator $C$ commutes with the generators $\left\{K_{0}, K_{ \pm}\right\}$of the algebra. In the $\mathrm{SU}(1,1)$ case, the Casimir operator turns out to be the $z$ component of the orbital angular momentum $C=L_{z}$, which is therefore left unchanged under the action of the squeezing operator $\left[U_{\alpha}^{\dagger}, L_{z}\right]=0$. This fact has been used in Sec. V, where the expected value of such Casimir operator is used as an order parameter that reveals the existence of a quantum phase transition.

$\mathrm{SU}(1,1)$ coherent states present several remarkable nonclassical properties, which attain an special interest in quantum optics since they can be engineered in experiments by means of a degenerate parametric amplifier. In the relativistic setting, they arise naturally as the eigenstates of a relativistic oscillator subjected to a magnetic field along the $z$ axis. In particular, we shall be interested in the following properties of such states, which have been extensively studied in Ref. [37]

$$
\begin{gathered}
\left\langle n_{r}\right\rangle_{\left|z, n_{l}\right\rangle}=\left(n_{l}+1\right) \operatorname{sh}^{2}|z|, \\
\left\langle n_{l}\right\rangle_{\left|z, n_{l}\right\rangle}=n_{l} \operatorname{ch}^{2}|z|+\operatorname{sh}^{2}|z|, \\
\left\langle n_{r}^{2}\right\rangle_{\left|z, n_{l}\right\rangle}=\left(n_{l}+1\right)^{2} \operatorname{sh}^{4}|z|+\left(1+n_{l}\right) \operatorname{ch}^{2}|z| \operatorname{sh}^{2}|z|, \\
\left\langle n_{l}^{2}\right\rangle_{\left|z, n_{l}\right\rangle}=n_{l}^{2} \operatorname{ch}^{4}|z|+\operatorname{sh}^{4}|z|+\left(1+3 n_{l}\right) \operatorname{ch}^{2}|z| \operatorname{sh}^{2}|z|,
\end{gathered}
$$

for the $\mathrm{SU}(1,1)$ coherent states in the left-handed regime, where $\operatorname{sh}|z|:=\sinh |z|$, and $\operatorname{ch}|z|:=\cosh |z|$, and we use the chiral number operators $n_{r}:=a_{r}^{\dagger} a_{r}$, and $n_{l}:=a_{l}^{\dagger} a_{l}$. Analogously, the corresponding coherent states in the right-handed regime fulfill the following:

$$
\begin{gathered}
\left\langle\tilde{n}_{l}\right\rangle_{\left|z, \tilde{n}_{r}\right\rangle}=\left(1+\tilde{n}_{r}\right) \operatorname{sh}^{2}|\tilde{z}|, \\
\left\langle\tilde{n}_{r}\right\rangle_{\left|\tilde{z}, \tilde{n}_{r}\right\rangle}=\tilde{n}_{r} \operatorname{ch}^{2}|\tilde{z}|+\operatorname{sh}^{2}|\tilde{z}|, \\
\left\langle\tilde{n}_{l}^{2}\right\rangle_{\left|\tilde{z}, \tilde{n}_{r}\right\rangle}=\left(\tilde{n}_{r}+1\right)^{2} \operatorname{sh}^{4}|\tilde{z}|+\left(1+\tilde{n}_{r}\right) \operatorname{ch}^{2}|\tilde{z}| \operatorname{sh}^{2}|\tilde{z}|, \\
\left\langle\tilde{n}_{r}^{2}\right\rangle_{\left|\tilde{z}, \tilde{n}_{r}\right\rangle}=\tilde{n}_{r}^{2} \operatorname{ch}^{4}|\tilde{z}|+\operatorname{sh}^{4}|\tilde{z}|+\left(1+3 \tilde{n}_{r}\right) \operatorname{ch}^{2}|\tilde{z}| \operatorname{sh}^{2}|\tilde{z}|,
\end{gathered}
$$

where $\operatorname{sh}|\tilde{z}|:=\sinh |\tilde{z}|$, and $\operatorname{ch} \tilde{r}:=\cosh |\tilde{z}|$, and we use the chiral number operators $\tilde{n}_{r}:=\tilde{a}_{r}^{\dagger} \tilde{a}_{r}$, and $\tilde{n}_{l}:=\tilde{a}_{l}^{\dagger} \tilde{a}_{l}$. These expressions (A6) and (A7) are extremely useful for the study of the relativistic phonon statistics in Sec. V.
[1] S. Sachdev, Quantum Phase Transitions (Cambridge University Press, Cambridge, England, 2000).

[2] N. Lambert, C. Emary, and T. Brandes, Phys. Rev. Lett. 92, 073602 (2004).

[3] C. Emary and T. Brandes, Phys. Rev. Lett. 90, 044101 (2003).

[4] C. C. Gerry, Phys. Rev. A 37, 3619 (1988).

[5] C. C. Gerry and J. Kiefer, Phys. Rev. A 41, 27 (1990).

[6] A. P. Hines, C. M. Dawson, R. H. McKenzie, and G. J. Milburn, Phys. Rev. A 70, 022303 (2004).

[7] M. Moshinsky and A. Szczepaniak, J. Phys. A 22, L817 (1989).

[8] D. Ito, K. Mori, and E. Carrieri, Nuovo Cimento A 51, 1119 (1967).

[9] W. Greiner, Relativistic Quantum Mechanics: Wave Equations (Springer, Berlin, 2000).

[10] A. Bermudez, M. A. Martin-Delgado, and E. Solano, Phys. Rev. A 76, 041801(R) (2007).

[11] A. Bermudez, M. A. Martin-Delgado, and E. Solano, Phys. Rev. Lett. 99, 123602 (2007)

[12] R. Banerjee and S. Gosh, J. Phys. A 31, L603 (1998).

[13] M. M. Horta Barreira and C. Wotzasek, Phys. Rev. D 45, 1410 (1992).

[14] G. V. Dunne, R. Jackiw, and C. A. Trugenberger, Phys. Rev. D 41, 661 (1990).
[15] E. T. Jaynes and F. W. Cummings, Proc. IEEE 51, 89 (1963)

[16] D. Leibfried, R. Blatt, C. Monroe, and D. Wineland, Rev. Mod. Phys. 75, 281 (2003).

[17] Note that these creation-annihilation operators do not represent normal modes since they do not commute with each other, but rather fulfill $\left[\underset{\sim}{a_{i}}, \widetilde{a}_{j}\right]=\delta_{i j} \mu,\left[a_{i}, \widetilde{a}_{j}^{\dagger}\right]=\delta_{i j} \tilde{\mu}$, where $\mu=: \frac{1}{2}\left(\frac{\Delta}{\tilde{\Delta}}-\frac{\tilde{\Delta}}{\Delta}\right)$ and $\tilde{\mu}=\frac{1}{2}\left(\frac{\Delta}{\tilde{\Delta}}+\frac{\tilde{\Delta}}{\Delta}\right)$.

[18] P. Meystre and M. Sargent III, Elements of Quantum Optics (Springer, Berlin, 2007).

[19] S. M. Barnett and P. L. Knight, J. Opt. Soc. Am. B 2, 467 (1985).

[20] A. Osterloh, L. Amico, G. Falci, and R. Fazio, Nature (London) 416, 608 (2002).

[21] G. Vidal, J. I. Latorre, E. Rico, and A. Kitaev, Phys. Rev. Lett. 90, 227902 (2003).

[22] M. Nielsen and I. Chuang, Quantum Computation and Quantum Information (Cambridge University Press, Cambridge, 2000).

[23] A. Galindo and M. A. Martin-Delgado, Rev. Mod. Phys. 74, 347 (2002)

[24] C. H. Bennett, D. P. DiVincenzo, J. A. Smolin, and W. K. Wootters, Phys. Rev. A 54, 3824 (1996).

[25] H. Casini, C. D. Fosco, and M. Huerta, J. Stat. Mech.: Theory Exp. (2005), P007. 
[26] P. Calabrese and J. Cardy, J. Stat. Mech.: Theory Exp. (2004), P06002.

[27] L. Amico, R. Fazio, A. Osterloh, and V. Vedral, e-print arXiv:quant-ph/0703044.

[28] M. B. Plenio and S. Virmani, Quantum Inf. Comput. 7, 1 (2007).

[29] J. Eisert and M. B. Plenio, Int. J. Quantum Inf. 1, 479 (2003).

[30] G. S. Agarwal, Phys. Rev. A 3, 828 (1971).

[31] A. Serafini, F. Illuminati, and S. De Sienna, J. Phys. B 37, L21 (2004).
[32] J. I. Cirac, A. S. Parkins, R. Blatt, and P. Zoller, Phys. Rev. Lett. 70, 556 (1993).

[33] J. Janszky and Y. Y. Yushin, Opt. Commun. 59, 151 (1986).

[34] D. J. Heinzen and D. J. Wineland, Phys. Rev. A 42, 2977 (1990).

[35] R. J. Glauber, Phys. Rev. 130, 2529 (1963); 131, 2766 (1963).

[36] A. M. Perelomov, Commun. Math. Phys. 26, 222 (1972).

[37] C. C. Gerry, J. Opt. Soc. Am. B 8, 685 (1991).

[38] L. Gilles and P. L. Knight, J. Mod. Opt. 39, 1411 (1992). 